\title{
Spatio-temporal Patterns of High Mountain Asia's Snowmelt Season Identified with an Automated Snowmelt Detection Algorithm, 1987-2016
}

\author{
Taylor Smith ${ }^{1}$, Bodo Bookhagen ${ }^{1}$, Aljoscha Rheinwalt ${ }^{1}$ \\ ${ }^{1}$ Institute of Earth and Environmental Sciences, Universität Potsdam, Germany
}

Corresponding author:

Taylor Smith

Institute of Earth and Environmental Sciences

Universität Potsdam

Potsdam-Golm 14476, Germany

Email: tasmith@uni-potsdam.de 


\section{Properties of Passive Microwave Sensors}

Table S 1 - Characteristics of PM sensors. Temporal coverage, number of orbits, and processing algorithms.

\begin{tabular}{llll}
\hline Satellite & Temporal Coverage & $\begin{array}{l}\text { Number of Orbits Used } \\
\text { (Descending/Ascending) }\end{array}$ & $\begin{array}{l}\text { Processing } \\
\text { Level/Algorithm }\end{array}$ \\
\hline SSMI & $\begin{array}{l}\text { Aug } 1987 \text { - Apr 2009 } \\
\text { (22 years) }\end{array}$ & FCDR V07 \\
SSMI/S & $\begin{array}{l}\text { Jan 2008 - Apr 2015 } \\
\text { (7 years) }\end{array}$ & $41,896 / 41,896$ & FCDR V07 \\
AMSR-E & $\begin{array}{l}\text { May 2002 - Oct } 2011 \\
\text { (9 years })\end{array}$ & $49,083 / 49,079$ & L1B \\
AMSR2 & $\begin{array}{l}\text { Jul 2012 - Oct 2016 } \\
\text { (4 years) }\end{array}$ & $28,510 / 28,506$ & L1R \\
GPM & $\begin{array}{l}\text { Feb 2014 - Oct 2016 } \\
\text { (2.5 years) }\end{array}$ & $7,359 / 7,359$ & L1B \\
& & &
\end{tabular}




\section{Flowchart of Melt Tracking Algorithm}

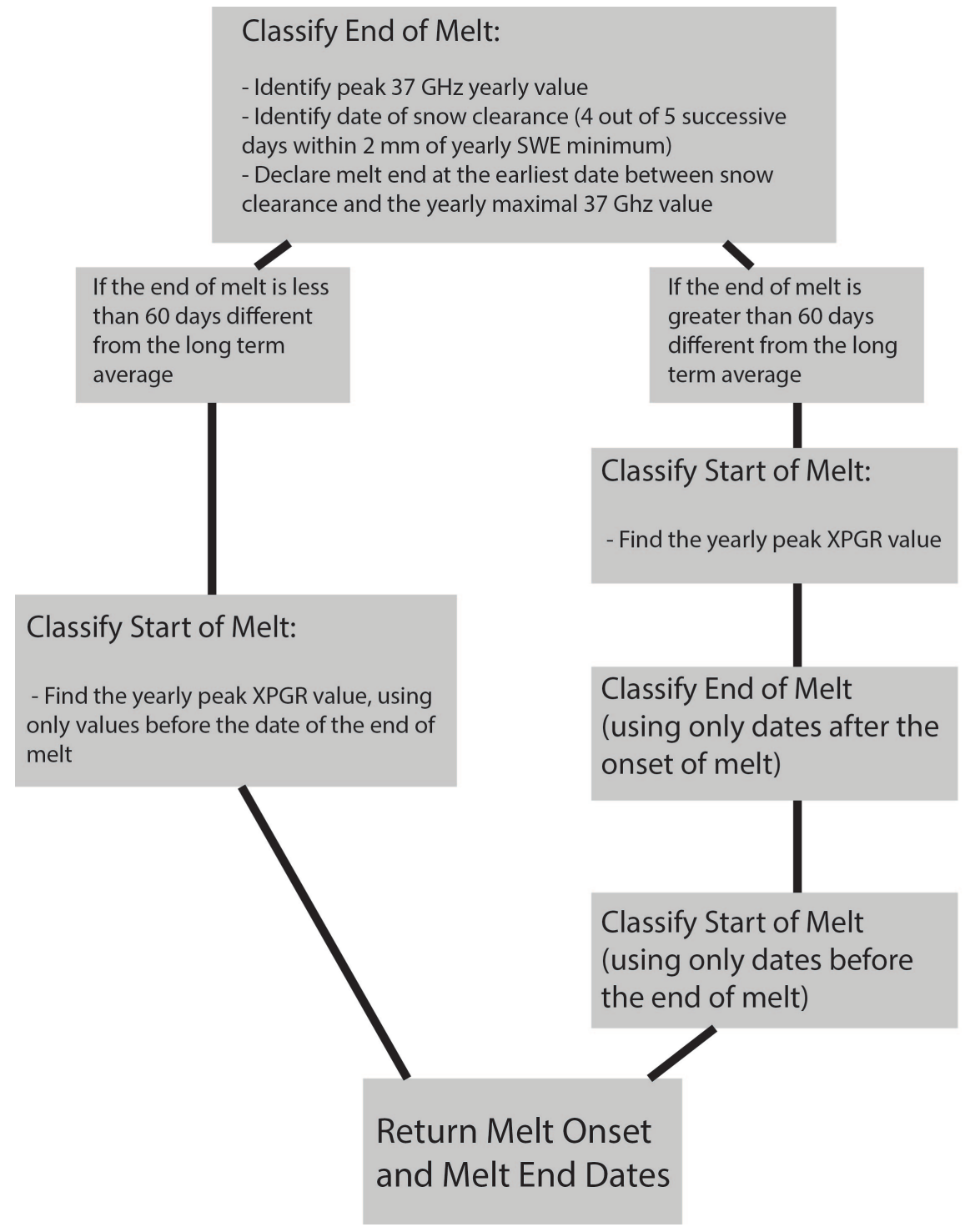

Figure S 1 - Flowchart illustrating the steps for the melt tracking algorithm. 


\section{Linear Matching Regression Parameters}




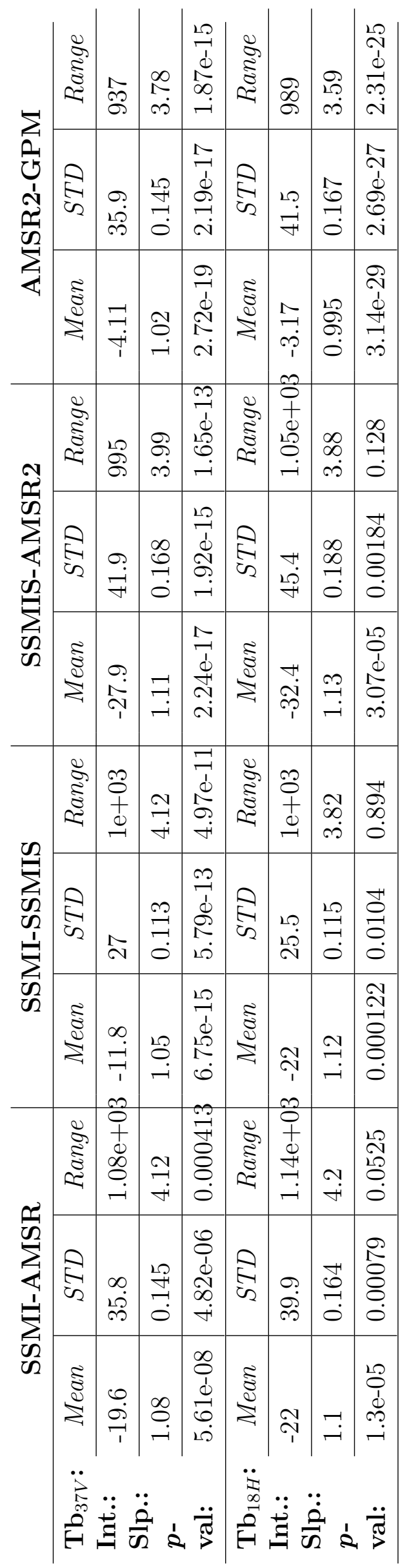




\section{Hierarchical Clustering Metrics}

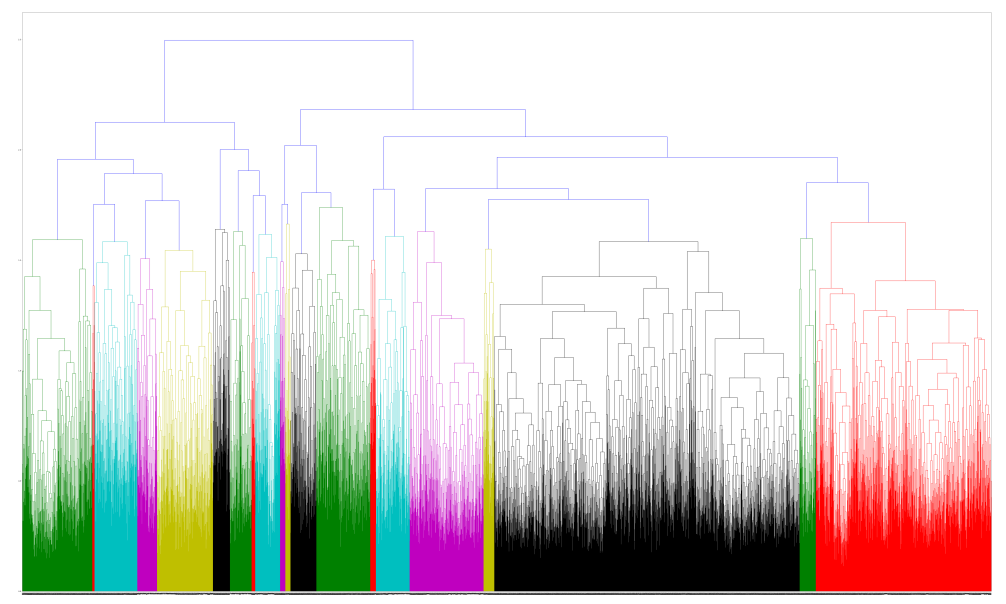

Figure S 2 - Hierarchical clustering Dendrogram.
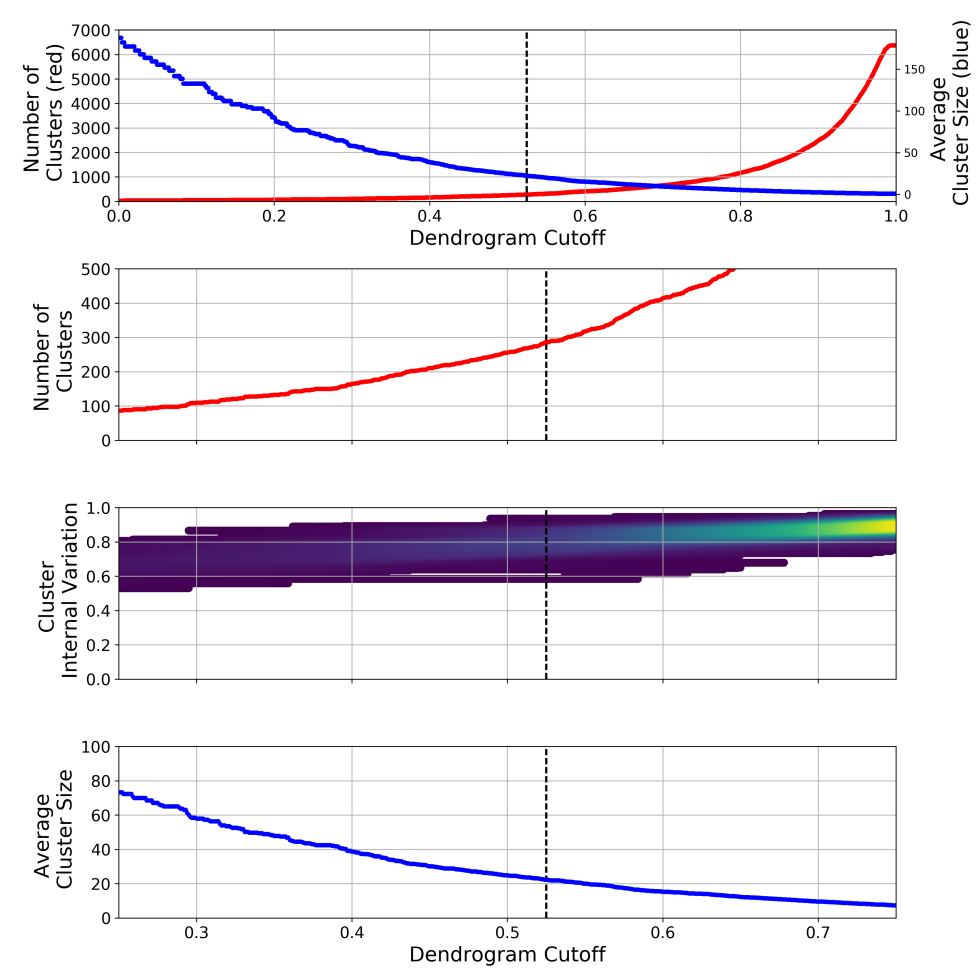

Figure S 3 - Metrics used to choose the hierarchical clustering distance threshold. Chosen threshold shown on each chart in black. 


\section{Impact of Analysis Timeframe}

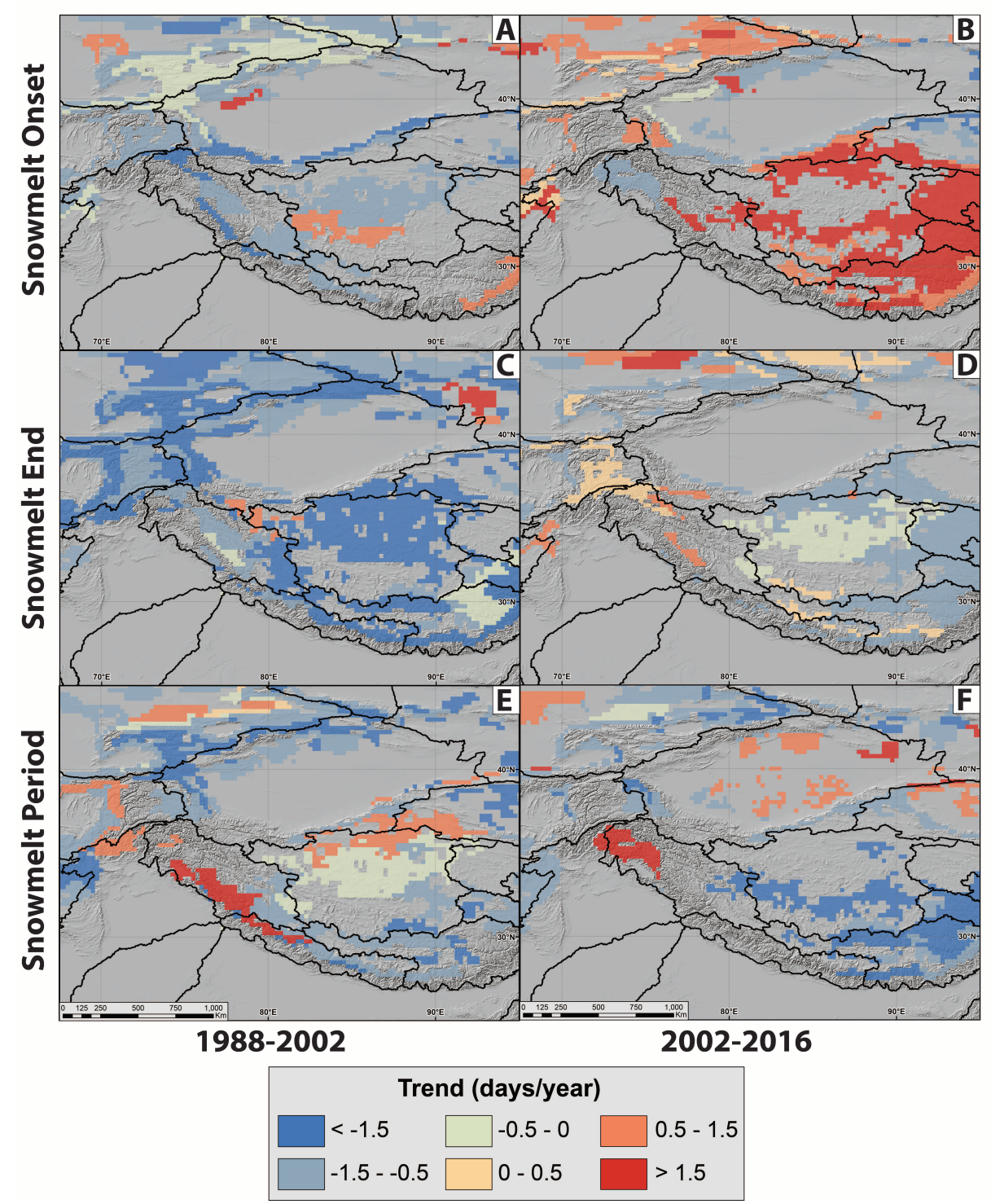

Figure S 4 - Impact of analysis timeframe on (A, B) snowmelt onset, (C,D) snowmelt end, and $(\mathrm{E}, \mathrm{F})$ snowmelt period. The onset date shows a reversal of trend in many regions, from negative to positive. Some snowmelt end dates have also reversed in trend. 